\title{
Stopper Container Closure
}

National Cancer Institute

\section{Source}

National Cancer Institute. Stopper Container Closure. NCI Thesaurus. Code C96139.

Object used to plug opening of container. 